\title{
Intensity-based framework and penalty formu- lation of optimal stopping problems
}

\author{
Min Dai
}

National University of Singapore

Yue Kuen Kwok

Hong Kong University of Science and Technology

\section{Hong You}

National University of Singapore

\begin{abstract}
Financial derivatives commonly contain pre-mature termination clauses, which are embedded rights held by the holder or writer. Well known examples of these stopping rights include the early exercise right in American options, the callable right in callable securities and the prepayment right in mortgage loans. In this paper, we show how to model the mortgagor's prepayment in mortgage loans and the issuer's call in the American warrant as an event risk using the intensity based approach, where the propensity of prepayment or calling is modeled by the intensity of a Poisson process. We illustrate that the corresponding pricing formulation resembles the penalty approximation approach commonly used in the solution of the linear complementarity formulation of an optimal stopping problem. We obtain several theoretical results on the prepayment strategies of mortgage loans and calling polices of American warrants. We also propose robust second order accurate numerical schemes for solving the penalty formulation of an optimal stopping problem.
\end{abstract}

Keywords: linear complementarity formulation, mortgage prepayment, callable feature, intensity approach, penalty method, event risk

\section{Introduction}

Mortgage loans and bond contracts are both debt instruments, except that the repayment of the principal in a mortgage loan is amortized over the life 
of the mortgage while the bond par is usually paid in full at maturity of the bond. The mortgagor plays a similar role as the bond issuer since both owe the scheduled stream of cash flows on their liabilities. Most mortgage loans contain the embedded prepayment privilege that gives the mortgagor the right to terminate the contract prematurely by paying the remaining principal plus any applicable transaction costs. The studies on the behaviors of prepayment and mortgage termination have been well explored in the literature in the past decades. Prepayment models that include the consideration of burnout effects and macro-economic factors have been proposed for the valuation of different types of mortgage backed securities (Deng et al., 2000; McConnell and Singh, 1994; Schwartz and Torous, 1992; Stanton, 1995). In callable bonds and other types of callable derivative securities, the embedded callable right entitles the issuer to recall the derivative by paying a pre-set cash amount (call price). There may be some imposed constraints on the calling provision, like the soft and hard call requirements and notice period requirement (Lau and Kwok, 2004). The discussion of the optimal calling policies of callable American warrants and convertible bonds can be found in the papers by Kwok and Wu (2000) and Dai and Kwok (2007). In essence, both the prepayment and callable rights limit the market value of future cash flow liabilities through early termination of the contract. This is achieved through an exchange of future liabilities by an upfront single payment.

The mortgagor's prepayment in mortgage loans and issuer's callable feature in callable securities are vivid examples of pre-mature termination clauses that are commonly found in financial derivatives. Assuming that the execution of these rights is optimally chosen by the writer or buyer of these securities, the modeling of these early termination clauses can be formulated as optimal stopping problems. By solving the linear complementarity formulation of the pricing model, the optimal stopping rule and derivative price are obtained simultaneously. However, numerous empirical studies have shown that in general these rights would not be exercised optimally as dictated by the optimal stopping rules. Market frictions, corporate finance considerations and other factors may affect the "rational" behavior of exercising the embedded right of pre-mature termination.

The intensity based approach has been commonly used in credit risk modeling to model the arrival of a default event. A similar intensity based framework has been adopted by Carr and Linetsky (2001) and Szimayer (2004) in the valuation of executive stock options subject to potential early departure of the executive, and by Goncharov (2006) in the valuation of 
mortgage contracts. Also, Szimayer (2005) studies the valuation of American options in the presence of event risk by modeling the arrival of event risk as the first jump time of a Cox process.

The penalty method is a well known approximation approach for solving the linear complementarity formulation of an optimal stopping problem (Friedman, 1982). Forsyth and Vetzal (2002) propose an implicit finite difference scheme for valuing American options using the penalty approximation. Their scheme manages to achieve second order rate of convergence by using variable sized time steps. A similar penalty approximation approach has been used to price American options whose underlying asset process is modeled by the stochastic volatility model (Zvan et al., 1998) or jump diffusion model (d'Halluin et al., 2004). In addition, Khaliq et al. (2006) develop adaptive $\theta$-methods for solving the penalty formulation of pricing models of one-asset and two-asset American options.

In this paper, we present a concise mathematical framework of modeling the mortgagor's prepayment in mortgage loans and the issuer's call in American warrant as an event risk and model the propensity of event arrival by the intensity of a Poisson process. We show that the intensity of calling or prepayment can be visualized as the penalty parameter in the penalty approximation. Without any surprise, infinite value of intensity represents the scenario where the right is exercised following the optimal stopping rule. This is consistent with the mathematical property that the penalty approximation of the linear complementarity formulation becomes "exact" when the value of the penalty parameter is taken to be infinity. Our contributions also include the construction of two versions of finite difference schemes for solving the penalty formulation of an optimal stopping problem. Our numerical tests reveal that the proposed numerical schemes demonstrate quadratic rate of convergence. This paper is organized as follows. In Section 2, we review the rational prepayment model proposed by Stanton (1995). The Stanton model allows for prepayment under exogenous and endogenous causes. By modeling the arrival of the prepayment event by a Poisson process, we derive the governing differential equation of the continuous version of Stanton's model. We then show how the differential equation formulation can be interpreted as the penalty approximation of the linear complementarity formulation of an optimal stopping problem. We analyze the monotonicity property of the mortgage value with respect to the intensity of prepayment. We also examine the impact of the transaction costs on the boundary that separates the prepayment region and non-prepayment region. In Section 3, we propose 
two versions of second order finite difference schemes for solving the penalty formulation of an optimal stopping problem. Numerical tests were performed to demonstrate the quadratic rate of convergence of the numerical schemes. In Section 4, we model the propensity of the issuer's calling of an American warrant by the intensity of a Poisson process. The impact of the intensity of calling on the optimal holder's exercise policy of the American warrant is analyzed. We end the paper with a summary and concluding remarks in the last section.

\section{Intensity-based approach of pricing mort- gage loans with prepayment option}

In this section, we concentrate on the pricing of a single mortgage loan by modeling the prepayment decision process of the mortgagor. In Stanton's prepayment model (1995), the arrival of prepayment event is modeled using the intensity based approach. His model allows two commonly observed "non-optimal" behaviors: mortgagors may prepay when it is not financially optimal to do so and delay refinancing even if prepayment is financially more beneficial.

Under the full "optimality" assumption, each mortgagor minimizes the market value of the mortgage liabilities and acts rationally to exercise the prepayment right. Let $L$ denote the present value of the mortgagor's liabilities and $P(t)$ be the remaining outstanding principal of the mortgage loan. Transaction costs are incurred upon prepayment. Here, the transaction costs should be interpreted in a broader sense. Besides the actual monetary costs, they also include the inconvenience costs, like the burden of going through the whole prepayment procedure. In general, transaction costs should contain a fixed component and a proportional factor. In our model, we take the simplifying assumption of zero fixed cost component. Now, the transaction cost is assumed to be proportional to the outstanding principal, which can be written as $P(t) X$, where $X$ is the proportional factor of transaction cost. The total payout $\psi(t)$ by the mortgagor upon prepayment is then equal to $P(t)(1+X)$. As a remark, the pricing formulation presented below can incorporate a non-zero fixed cost component in a relatively straightforward manner. The full optimality assumption would lead to the following simple prepayment decision rule: prepay whenever $L>\psi(t)$ and not to do so 
otherwise.

In our mathematical setup, we assume the existence of a martingale pricing measure $Q$, implying the absence of arbitrage. The uncertainty of the economy is modeled by a filtered probability space $\left(\Omega, \mathcal{G},\left\{\mathcal{G}_{t}\right\}_{t \geq 0}, Q\right)$, where the $\sigma$-algebra $\mathcal{G}_{t}$ represents all observations available to the mortgagor at time $t, \Omega$ is the sample space of all outcomes and $Q$ is a risk neutral measure on $\mathcal{G}$, where $\mathcal{G} \supseteq \cup_{t \geq 0} \mathcal{G}_{t}$. In our continuous-time diffusion state process setting, we take the interest rate as the single stochastic state variable in the pricing model. Let $\tau$ denote the prepayment time of the mortgage loan, which is a positive stopping time on this filtered probability space. Let $\gamma_{t}$ denote the intensity of the random prepayment time $\tau$, then $\gamma_{t}$ is a $\mathcal{G}_{t}$-adapted intensity process. We consider another filtration $\mathcal{F}_{t}$, which is the natural filtration generated by the interest rate process. Since the prepayment decision is not driven by the interest rate movement alone, $\tau$ is not an $\mathcal{F}_{t^{-}}$stopping time.

Following the modeling of prepayment behaviors as postulated by Stanton (1995), a mortgagor may prepay his mortgage loan either for exogenous or endogenous reasons. We refer to prepayment due to migration, divorce, etc., those not driven by interest rate considerations, as exogenous. The arrival of an exogenous prepayment is modeled as a Poisson process with constant intensity $\lambda$. On the other hand, a mortgagor may consider to refinance when $L>\psi(t)$. Such a prepayment decision is said to be endogenous. The endogenous prepayment is also modeled by a Poisson process with intensity $\rho \mathbf{1}_{\{L>\psi(t)\}}$, where $\rho$ is a constant, reflecting the fact that the intensity of endogenous prepayment is zero when $L \leq \psi(t)$. The prepayment time $\tau$ is the minimum of these two independent random times, so the intensity $\gamma_{t}$ of $\tau$ is simply the sum of their intensities. The intensity $\gamma_{t}$ has dependence on $L$, which can be expressed as

$$
\gamma_{t}=\left\{\begin{array}{ll}
\lambda & \text { if } L \leq \psi(t) \\
\lambda+\rho & \text { if } L>\psi(t)
\end{array} .\right.
$$

\section{Partial differential equation formulation}

Under the risk neutral measure $Q$, the dynamics of the stochastic short rate $r_{t}$ is assumed to be governed by the Ito process

$$
d r=\mu_{r}(r, t) d t+\sigma_{r}(r, t) d Z,
$$

where $\mu_{r}$ is the drift, $\sigma_{r}$ is the volatility and $d Z$ is the differential of the standard Wiener process. Let $c(t)$ denote the continuous amortized cash 
flows paid by the mortgagor throughout the contract until termination. In practice, mortgage payments are made at discrete times. This corresponds to

$$
c(t)=c_{i} \delta\left(t-t_{i}\right) \quad i=1,2, \cdots, n,
$$

where $c_{i}$ is the discrete payment made at time $t_{i}$ and $\delta\left(t-t_{i}\right)$ is the Dirac function. We write $L(r, t)$ as the value of the mortgage liabilities and $\gamma(L)$ as the intensity of the prepayment time with dependence on $L$. The governing equation for $L(r, t)$ can be derived from the following relation

$$
r L d t=E_{t}[d L+c(t) d t],
$$

where $E_{t}$ is the expectation under $Q$ conditional on the filtration $\mathcal{G}_{t}$. Provided that prepayment has not occurred up to time $t$, the conditional probability that prepayment occurs over $(t, t+d t)$ is $\gamma(L) d t$. Upon occurrence of prepayment, there is a change in liability value of amount $\psi(t)-L$. By Ito's lemma, we have

$$
\begin{aligned}
r L d t= & \frac{\partial L}{\partial t} d t+\mu_{r} \frac{\partial L}{\partial r} d t+\frac{\sigma_{r}^{2}}{2} \frac{\partial^{2} L}{\partial r^{2}} d t \\
& +\gamma(L)[\psi(t)-L] d t+c(t) d t .
\end{aligned}
$$

The differential equation for $L(r, t)$ is then obtained as follows

$$
\frac{\partial L}{\partial t}+\mu_{r} \frac{\partial L}{\partial r}+\frac{\sigma_{r}^{2}}{2} \frac{\partial^{2} L}{\partial r^{2}}-[r+\gamma(L)] L+c(t)+\gamma(L) \psi(t)=0 .
$$

Based on the intensity $\gamma_{t}$ defined in eq. (2.1), the above governing equation can be succinctly expressed as

$$
\begin{aligned}
& \frac{\partial L}{\partial t}+\mu_{r} \frac{\partial L}{\partial r}+\frac{\sigma_{r}^{2}}{2} \frac{\partial^{2} L}{\partial r^{2}}-(r+\lambda) L+c(t)+\lambda \psi(t) \\
= & \rho \max (L-\psi(t), 0) .
\end{aligned}
$$

For a finite value of $\rho$, the penalty term $\rho \max (L-\psi(t), 0)$ models a suboptimal policy of endogenous prepayment. The governing equation is seen to be a mildly non-linear differential equation due to the presence of the penalty term. The limiting case of $\rho \rightarrow \infty$ corresponds to the policy that prepayment becomes immediate when $L$ reaches $\psi(t)$ from below.

The full description of the pricing model requires the prescription of the auxiliary conditions. At maturity $T$, we have $L(r, T)=0$. As for the boundary conditions, it is obvious that $L(r, t) \rightarrow 0$ as $r \rightarrow \infty$. However, it is 
quite tricky to apply the boundary condition at the left end of the domain for most short rate models. In the numerical solution of the pricing model using the finite difference approach (see Sec. 3), the numerical calculations of the value function are performed within a finite numerical domain. The discretization of the differential equation is performed at the left and right boundary node points using one-sided difference operators to approximate the differential operators. In this way, the artificial imposition of numerical boundary conditions at the boundary nodes is avoided.

There is a distinction between the value of liability to the mortgagor $L(r, t)$ and the fair value of the mortgage $M(r, t)$. The difference arises since the mortgagor pays $P(t)(1+X)$ at the time of prepayment but the value of the mortgage loan equals the outstanding principal $P(t)$ upon prepayment. The mortgage value is not simply given by the discounted expected value of the cash flow $c(t)$ since prepayment may occur. By following a similar argument as above and replacing the payment term $\psi(t)$ in eq. (2.3) by $P(t)$, the governing differential equation for $M(r, t)$ is deduced to be

$$
\begin{aligned}
& \frac{\partial M}{\partial t}+\mu_{r} \frac{\partial M}{\partial r}+\frac{\sigma_{r}^{2}}{2} \frac{\partial^{2} M}{\partial r^{2}}-(r+\lambda) M+c(t)+\lambda P(t) \\
= & \rho[M-P(t)] \mathbf{1}_{\{L>\psi(t)\}} .
\end{aligned}
$$

Given the known solution to $L(r, t)$, the above differential equation for $M(r, t)$ is linear.

The outstanding principal $P(t)$ can be obtained by solving the following differential equation

$$
c(t) d t=-d P(t)+m(t) P(t) d t
$$

where $m(t)$ is the deterministic mortgage rate. Using the initial condition: $P(0)=P_{0}$. The solution for $P(t)$ is easily found to be

$$
P(t)=P_{0} e^{\int_{0}^{t} m(u) d u}-\int_{0}^{t} c(s) e^{\int_{s}^{t} m(u) d u} d s .
$$

The relation between $c(t)$ and $m(t)$ can be established by observing the terminal condition: $P(T)=0$. If the amortized cashflow is taken to be independent of time, then the constant rate of cashflow $c$ is related to the fixed mortgage rate $m_{0}$ (set at initiation) by

$$
c=\frac{m_{0}}{1-e^{-m_{0} T}} P_{0} .
$$




\section{Limit of infinite value of intensity parameter}

Assuming the mortgagor to be fully optimal, the optimal endogenous strategy is to exercise the prepayment right immediately when the liability $L$ rises to the level $\psi(t)$. This would mean an infinite value for the intensity $\rho$. The mortgage pricing model then becomes an optimal stopping problem. Let $\mathcal{L}$ denote the operator defined as

$$
\mathcal{L}=\mu_{r} \frac{\partial}{\partial r}+\frac{\sigma_{r}^{2}}{2} \frac{\partial^{2}}{\partial r^{2}}-(r+\lambda)
$$

Let $L_{\text {opt }}$ denote the mortgagor's liability under the optimality condition (that is, $\rho \rightarrow \infty$ ). The linear complementarity (variational inequalities) formulation of the optimal stopping problem is given by

$$
\begin{gathered}
\frac{\partial L_{o p t}}{\partial t}+\mathcal{L} L_{o p t}+c(t)+\lambda \psi(t) \geq 0 \\
L_{o p t}-\psi(t) \leq 0 \\
{\left[\frac{\partial L_{o p t}}{\partial t}+\mathcal{L} L_{o p t}+c(t)+\lambda \psi(t)\right]\left[L_{o p t}-\psi(t)\right]=0 .}
\end{gathered}
$$

From the theory of variational inequalities of free boundary problems (Friedman, 1982), the differential equation formulation in eq. (2.4) can be visualized as the penalty approximation to the linear complementarity formulation in eq. (2.8). Here, the parameter $\rho$ in the penalty term has a specific financial interpretation in the prepayment model. It can be seen as the intensity of endogenous prepayment.

\section{Separating boundary}

Recall that a necessary condition for endogenous prepayment is given by $L>\psi(t)$. Obviously $\frac{\partial L}{\partial r} \leq 0$, so the critical threshold on the interest rate $r$ that separates $L>\psi(t)$ and $L<\psi(t)$ in the $(r, t)$-plane can be defined as

$$
r^{*}(t)=\min \{r(t) ; \quad L<\psi(t)\} .
$$

How does the separating boundary $r^{*}(t)$ depend on the intensity of prepayment and transaction cost? First, we would like to establish the monotonicity property of the liability value function with respect to the penalty parameter $\rho$ and other parameter functions. We then present the lemma that states the monotonicity property of $r^{*}(t)$ with respect to the proportional factor $X$ of transaction cost. 
Lemma 1

Let the liability value function $L_{i}, i=1,2$, be the solution to the following penalty formulation

$$
\left(\frac{\partial}{\partial t}+\mathcal{L}\right) L_{i}+f_{i}(t)=\rho_{i} \max \left(L_{i}-\psi, 0\right), \quad i=1,2 .
$$

Furthermore, the two liability value functions share the same set of initialboundary conditions. Here, $\rho_{1}$ and $\rho_{2}$ are penalty parameters, and $f_{1}(t)$ and $f_{2}(t)$ are source terms in the differential equation. Suppose $\rho_{1} \geq \rho_{2}>0$ and $f_{1}(t) \leq f_{2}(t)$ for all $t$, then the liability value functions $L_{1}$ and $L_{2}$ observe

$$
L_{1} \leq L_{2} \text { for all values of } \rho \text { and } t .
$$

The financial intuition behind the result in Lemma 1 is quite obvious. The liability value of mortgage 1 cannot be higher than that of mortgage 2 if the contract holder in mortgage 1 acts at least as optimally as the counterpart in mortgage $2\left(\rho_{1} \geq \rho_{2}\right)$ and the payments being made in the first mortgage are no more than those in the second marotgage $\left[f_{1}(t) \leq f_{2}(t)\right]$. The proof of Lemma 1 using the comparison principle in partial differential equation theory is presented in Appendix A. Using the monotonicity result established in Lemma 1, it becomes straightforward to show Lemma 2.

Lemma 2

If $X_{1}<X_{2}$, then $r^{*}\left(t ; X_{1}\right) \geq r^{*}\left(t ; X_{2}\right)$.

With a higher transaction cost, the interest rate has to be lowered further in order to increase the value of liability $L$ to the level of prepayment payout $\psi(t)$. The result is Lemma 2 is seen to agree with this financial intuition. The proof of Lemma 2 is presented in Appendix B.

In Figure 1, we show the plot of the separating boundary $r^{*}(t)$ against time to expiry $T-t$ with varying values of the penalty parameter $\rho$ and proportional transaction cost factor $X$. In our calculations, we use the same set of parameter values in the Cox-Ingersoll-Ross short rate model as those used by Stanton (1995). Here, the interest rate dynamics under $Q$ is given by

$$
d r=[0.29368(0.07935-r)+0.12165 r] d t+0.11425 \sqrt{r} d Z .
$$

The other parameter values are: $T=30, \lambda=0.3$ and $m_{0}=0.08$. The rate of amortized cashflow is assumed to be constant, so it can be obtained 
from Eq. (2.7). The curves in Figure 1 show the critical threshold value of interest rate $r^{*}(t)$ that separates the non-prepayment region in the above and the feasible prepayment region below. In the limiting case where $\rho$ assumes the infinite value, the separation boundary becomes the free boundary. In this case, the holder chooses to exercise once the interest rate falls to $r^{*}(t)$. Since $L$ is decreasing with respect to both $\rho, X$ and $r$, a lower value of $r^{*}(t)$ is resulted when $\rho$ or $X$ assumes a higher value. The plots of the separation boundaries in Figure 1 serve to verify the monotonicity properties of $r^{*}(t)$ with respect to $\rho$ and $X$ as deduced from Lemmas 1 and 2. Since the curves in Figure 1 intersect the horizontal time axis at positive value of time to expiry, we deduce that there exists a critical value of time to expiry such that the mortgage is never prepaid when the time to expiry is less than this critical value.

\section{Second order accurate numerical schemes for solving penalty formulation}

In this section, we propose two second order time accurate finite difference schemes for solving the penalty formulation presented in eq. (2.4). A similar numerical solution using the finite difference approach has been considered by Stanton (1995). He employs the Crank-Nicolson type discretization in the approximation of the differential operators but the non-linear penalty term is discretized explicitly. The second order accuracy of the Crank-Nicolson scheme cannot be achieved since the penalty term is evaluated explicitly.

We consider the construction of finite difference schemes for the following prototype equation, which is obtained from the penalty approximation of the linear complementarity formulation of the pricing model of an American option with the exercise payoff function $\phi(S)$ :

$$
\frac{\partial U}{\partial \tau}=\frac{\sigma^{2}}{2} S^{2} \frac{\partial^{2} U}{\partial S^{2}}+r S \frac{\partial U}{\partial S}-r U+\rho \max (\phi(S)-U, 0) .
$$

Here, the option value function $U(S, \tau)$ is a function of stock price $S$ and time to expiry $\tau$, where $\tau=T-t$, and $\sigma$ is the volatility of the stock price. In this section, we propose two modifications of the discretization of the penalty term in the Crank-Nicolson scheme proposed by Forsyth and Vetzal (2002). The objectives are to provide alternative schemes that exhibit second order 
convergence behavior without the adoption of variable sized time steps in the calculations.

Let $U_{j}^{n}$ denote the discrete numerical approximation to $U\left(S_{j}, \tau_{n}\right)$, where $S_{j}=S_{0}+j \Delta S, j=1,2, \cdots, N_{S}$, and $\tau_{n}=n \Delta \tau, n=1,2, \cdots, N_{\tau}$. Here, $\Delta S$ and $\Delta \tau$ are the stepwidth and time step, respectively, $N_{S}$ and $N_{\tau}$ are the total number of spatial and temporal grids, respectively. Define the spatial difference operator $\mathcal{L}_{h}$ by

$$
\mathcal{L}_{h} U_{j}^{n}=\frac{\sigma^{2}}{2} S_{j}^{2} \frac{U_{j+1}^{n}-2 U_{j}^{n}+U_{j-1}^{n}}{\Delta S^{2}}+r S_{j} \frac{U_{j+1}^{n}-U_{j-1}^{n}}{2 \Delta S}-r U_{j}^{n} .
$$

Forsyth and Vetzal (2002) propose the following Crank-Nicolson discretization of eq. (3.1) with an implicit treatment of the penalty term:

$$
U_{j}^{n+1}=U_{j}^{n}+\frac{\Delta \tau}{2} \frac{\mathcal{L}_{h} U_{j}^{n+1}+\mathcal{L}_{h} U_{j}^{n}}{2}+\xi^{n+1}\left[\phi\left(S_{j}\right)-U_{j}^{n+1}\right]
$$

where

$$
\xi^{n+1}=\left\{\begin{array}{ll}
\rho \Delta \tau & \text { if } \phi\left(S_{j}\right)>U_{j}^{n+1} \\
0 & \text { otherwise }
\end{array} .\right.
$$

Since the penalty term $\xi^{n+1}\left[\phi(S)-U_{j}^{n+1}\right]$ is non-linear, one has to solve a nonlinear system of algebraic equations at each time step to obtain $U_{j}^{n+1}$. Forsyth and Vetzal (2002) propose the generalized Newton iteration procedure for the numerical solution of the non-linear system. In their numerical calculations, they showed that a variable sized time steps approach should be adopted in order to guarantee second order accuracy of the numerical results.

Our two modified schemes involve some slight modification of the discretized penalty term in eqs. (3.2a,b). Unlike the Forsyth-Vetzal scheme, our schemes achieve second order accuracy of numerical results even when constant sized time steps are used in the calculations.

Scheme One

The penalty term is discretized at $\left(n+\frac{1}{2}\right)^{\text {th }}$ time level, so that it now becomes

$$
\xi^{n+\frac{1}{2}}\left[\phi\left(S_{j}\right)-\frac{U_{j}^{n+1}+U_{j}^{n}}{2}\right]
$$

where

$$
\xi^{n+\frac{1}{2}}= \begin{cases}\rho \Delta \tau & \text { if } \phi\left(S_{j}\right)>\frac{U_{j}^{n+1}+U_{j}^{n}}{2} \\ 0 & \text { otherwise }\end{cases}
$$


Since the penalty term is discretized in a semi-implicit manner, so iterations of Newton type are also required at each time step.

\section{Scheme Two}

The non-linearity in the penalty term disappears when we replace the implicit term $\left(U_{j}^{n+1}+U_{j}^{n}\right) / 2$ by the explicit term $\left(3 U_{j}^{n}-U_{j}^{n-1}\right) / 2$. Now, the solution for $U_{j}^{n+1}$ amounts to the solution of a linear tridiagonal system of algebraic equations. The discretized penalty term is given by

$$
\widehat{\xi}^{n+\frac{1}{2}}\left[\phi\left(S_{j}\right)-\frac{3 U_{j}^{n}-U_{j}^{n-1}}{2}\right],
$$

where

$$
\widehat{\xi}^{n+\frac{1}{2}}=\left\{\begin{array}{ll}
\rho \Delta \tau & \text { if } \phi\left(S_{j}\right)>\frac{3 U_{j}^{n}-U_{j}^{n-1}}{2} \\
0 & \text { otherwise }
\end{array} .\right.
$$

Note that Scheme Two is an explicit three-level scheme. As the discretized scheme does not lead to a non-linear equation for $U_{j}^{n+1}$, enhanced computational efficiency is achieved as Newton type of iterations at every time step is avoided. As part of the initiation procedure, one has to use an alternative two-level second order accurate scheme to obtain the numerical solution at the first time level. More detailed discussion on the construction and solution of various types of three-level finite difference schemes can be found in Thomas' text (1995).

Forsyth and Vetzal (2002) present a proof on the convergence of their penalty algorithm $(3.2 \mathrm{a}, \mathrm{b})$ based on an implicit discretization of the nonlinear penalty term. In our Scheme One, we adopt a semi-implicit treatment of the non-linear term. By following a similar approach of using the discrete maximum principle, we are able to establish the convergence of Scheme One. The outline of the proof is presented in Appendix C.

\section{Numerical calculations}

To compare the convergence behavior of the numerical results obtained using our Scheme One and the Forsyth-Vetzal scheme, we repeated similar pricing calculations of an American put option as reported in Forsyth and Vetzal's paper (2002) using constant sized time steps. The payoff of the American put option is $\phi(S)=\max (K-S, 0)$, where $K$ is the strike price. The numerical results are presented in Table 1. The parameter values used in the American put option are: $r=0.1, S=100, \tau=0.25, K=100$. For a second order time 
accurate scheme, we expect that the error of approximation is reduced by a factor of $1 / 4$ when the number of time steps is doubled. The numerical results obtained from our Scheme One demonstrate a quadratic rate of convergence. As demonstrated and explained in Forsyth-Vetzal's paper (2002), Scheme $(3.2 \mathrm{a}, \mathrm{b})$ does not exhibit second order accuracy when constant sized time steps are used. Forsyth and Vetzal show that in order to achieve second order rate of convergence in their scheme, variable sized time steps have to be adopted in the numerical calculations.

Both our proposed schemes and Stanton's scheme were applied to the numerical valuation of the liability value of a mortgage loan. Following Stanton's numerical approach, we apply the transformation: $y=\frac{1}{1+1.25 r}$ so as to transform the unbounded domain to a bounded domain. We define the mean-squared error by

$$
\sqrt{\sum_{i=1}^{10}\left[V\left(r_{i}, 0\right)-V^{\text {exact }}\left(r_{i}, 0\right)\right]^{2}}
$$

where $r_{i}=\frac{1-y_{i}}{12.5 y_{i}}, y_{i}=0.1,0.2, \cdots, 1.0$. The so-called "exact" solution is obtained by taking both the number of time steps and number of spatial steps to be 25,600 in the numerical calculations. We measured the mean squared errors and examined the rates of decrease of the mean squared errors with increasing number of time steps. In Table 2, we present the numerical results in a typical mortgage loan valuation. The parameter values used in our calculation are: $X=0.1, \rho=0.05, \lambda=0.3, T=30$, and $m_{0}=0.08$. From the values of the successive ratios of the mean squared errors, we deduce that both Scheme One and Scheme Two are second order time accurate while Stanton's Scheme is only first order time accurate (as revealed from the observation that the error of approximation in the numerical results obtained from Stanton's Scheme is halved when the number of time steps is doubled).

In Table 3, we show the numerical results of the liability value of a mortgage loan under varying values of the transaction cost factor $X$, intensity of exogenous payment $\lambda$ and penalty parameter $\rho$. In our calculations, we used $r=0.02$ and other parameter values were taken to be the same as those used to generate the plots in Figure 1. As expected, the liability value is an increasing function of $\lambda$ but decreasing with respect to both $X$ and $\rho$. When $\lambda$ assumes zero or small value, the liability value becomes less sensitive to change in $X$ and $\rho$. 


\section{American warrant subject to issuer's call- ing}

In this section, we consider the impact of the issuer's calling right on the optimal early exercise policy of an American warrant. Examples of callable American warrants that are traded in the financial markets can be found in Kwok and $\mathrm{Wu}$ (2000). The payoff of the American warrant upon exercise by the holder is the usual call option payoff: $\max \left(S_{\hat{t}}-K, 0\right)$, where $S_{\widehat{t}}$ is the stock price at the exercise time $\hat{t}$ and $K$ is the strike price. Upon calling by the issuer, the American warrant is terminated prematurely. We assume that the holder receives fixed dollar amount $R$ as rebate from the issuer upon calling. The calling may be visualized as an event of pre-mature termination. In this sense, the callable American warrant can be interpreted as an American option subject to event risk of early termination (Szimayer, 2005). As noted by Szimayer, the presence of calling risk influences the optimal exercise policy adopted by the warrant holder.

We would like to derive the formulation of the pricing model of an American warrant subject to the risk of pre-mature termination by the issuer's calling. Let $V(S, t)$ be the price function of the American warrant, where $S$ is the stock price. Let $\phi(S)$ be payoff upon exercise and $\psi(S)$ be the rebate received by the holder upon calling. Assume the arrival of calling by the issuer to be governed by a Poisson process with intensity $\rho \mathbf{1}_{\{V>\psi\}}$, where $\rho$ is a constant. The indicator function $\mathbf{1}_{\{V>\psi\}}$ is included since the issuer calls only when $V>\psi$. In our callable American warrant, we have the exercise payoff $\phi(S)=S-K$ and rebate $\psi(S)=R$.

\section{Complementarity formulation}

Under the risk neutral measure $Q$, the dynamics of the stock price are assumed to be governed by

$$
\frac{d S}{S}=(r-q) d t+\sigma d Z
$$

where $q$ is the constant dividend yield of the stock. By following a similar argument as that of the value of the liability mortgage loan in Sec. 2, we obtain the following governing equation for $V(S, t)$ in the continuation region where the warrant remains alive. We obtain

$$
r V d t=\left[\frac{\partial V}{\partial t}+\frac{\sigma^{2}}{2} S^{2} \frac{\partial^{2} V}{\partial S^{2}}+(r-q) S \frac{\partial V}{\partial S}\right] d t+\rho d t \max (V-\psi, 0)
$$


so that

$$
\frac{\partial V}{\partial t}+\frac{\sigma^{2}}{2} S^{2} \frac{\partial^{2} V}{\partial S^{2}}+(r-q) S \frac{\partial V}{\partial S}-r V+\rho \max (V-\psi, 0)=0 .
$$

In the stopping region where it is optimal for the holder to exercise the warrant, we have

$$
V(S, t)=\phi(S) .
$$

We write $\tau=T-t$, where $\tau$ is the time to expiry and let $\mathcal{L}_{w}$ be the differential operator defined by

$$
\mathcal{L}_{w}=\frac{\sigma^{2}}{2} S^{2} \frac{\partial^{2}}{\partial S^{2}}+(r-q) S \frac{\partial}{\partial S}-r
$$

the complementarity formulation for $V(S, \tau)$ is given by

$$
\min \left(\frac{\partial V}{\partial \tau}-\mathcal{L}_{w} V+\rho \max (V-\psi, 0), V-\phi\right)=0
$$

subject to the terminal condition

$$
V(S, 0)=\max (\phi(S), 0)
$$

The above complementarity formulation is non-linear due to the presence of the penalty term $\max (V-\psi, 0)$. Recall that in the Carr-Linetsky model of executive stock valuation, the intensity of the process of event arrival does not depend on the value function, so their differential equation formulation remains linear (Carr and Linetsky, 2000). The numerical solution to the complementarity formulation $(4.4 \mathrm{a}, \mathrm{b})$ can be achieved using either scheme One or Scheme Two proposed in Sec 3, in combination with the dynamic programming procedure for the incorporation of the constraint: $V \geq \phi$ into the numerical calculations. This is like usual American option calculations in lattice tree calculations where the maximum of continuation value and exercise payoff are taken at each computational node.

Recall that the intensity of the Poisson process that models the occurrence of the issuer's call is given by $\rho \mathbf{1}_{\{V \geq \psi\}}$. In the limit $\rho \rightarrow \infty$, the arrival of the issuers call is immediate whenever $V$ reaches $\psi$ from below. In the literature, the so-called "delayed call" phenomena refer to the observation that the issuers may defer their calling even when the value of the callable derivative $V$ goes beyond the call rebate payment $\psi$ (Dai and Kwok, 2007). 
Thus, the case of " $\rho \rightarrow \infty$ " corresponds to the "no delayed call" scenario. For finite value of $\rho$, the pricing formulation becomes the "two-obstacle" problem with the upper obstacle function $\psi(S)$ and lower obstacle function $\phi(S)$. The complementarity formulation $(4.4 \mathrm{a}, \mathrm{b})$ now becomes linear and it is given by (Dai and Kwok, 2007)

$$
\min \left(\max \left(\frac{\partial V}{\partial \tau}-\mathcal{L}_{w} V, V-\psi\right), V-\phi\right)=0 .
$$

Now, the pricing model becomes the double obstacle problem since $V$ is bounded from above by $\psi$ and below by $\phi$. The interaction of the optimal calling and exercise policies of the American warrant under optimality of calling has been discussed by Dai and Kwok (2006).

In the simple case of $\psi=R$ and $\phi=S-K$, the optimal issuer's calling and holder's exercise policies are given by (Kwok and Wu, 2000)

$$
S^{*}(\tau)=\min \left(\widetilde{S}^{*}(\tau), K+R\right),
$$

where $S^{*}(\tau)$ denotes the optimal exercise price at which the warrant is either optimally called by the issuer or exercised by the holder. Here, $\widetilde{S}^{*}(\tau)$ denotes the optimal exercise price of the usual American call option. Kwok and Wu (2000) show that there is a critical value $\tau^{*}$ of the time to expiry at which

$$
\widetilde{S}^{*}\left(\tau^{*}\right)=K+R,
$$

and

$$
S^{*}(\tau)=\left\{\begin{array}{ll}
\widetilde{S}^{*}(\tau) & \text { when } \tau \leq \tau^{*} \\
K+R & \text { when } \tau>\tau^{*}
\end{array} .\right.
$$

The above property on $S^{*}(\tau)$ stems from the observation that the price of an American call option does not exceed $R$ when $\tau \leq \tau^{*}$.

When the arrival of calling is modeled as a Poisson process with intensity parameter $\rho$, we would like to examine the optimal exercise price $S_{\rho}^{*}(\tau)$ at which it is optimal for the holder to exercise. In Figure 2, we plot $S_{\rho}^{*}(\tau)$ against $\tau$ for varying values of intensity $\rho$. The parameter values used in the calculations are: $K=100, r=0.02, q=0.04, \sigma=0.3, T=2$ and $R=130$. Interestingly, it is observed from Figure 2 that

$$
S_{\rho}^{*}(\tau)=\widetilde{S}^{*}(\tau) \quad \text { for } \quad \tau \leq \tau^{*}
$$

and $S_{\rho}^{*}(\tau)$ is a decreasing function of $\rho$ when $\tau>\tau^{*}$. 
The monotonically decreasing property of $S_{\rho}^{*}(\tau)$ with respect to $\rho$ can be inferred easily from the monotonically decreasing property of the value function $V(S, \tau ; \rho)$ with respect to $\rho$. That is, a callable American warrant has a higher value if the issuer adopts the calling policy that deviates more from optimality. In particular, we have $V(S, \tau ; \rho) \geq V(S, \tau ; \infty)$ for any finite value of $\rho$. To show eq. (4.8), we argue that when $\tau \leq \tau^{*}$, the issuer would never call the American warrant since

$$
V(S, \tau ; \rho) \leq C_{A}(S, \tau) \leq R, \quad \tau \leq \tau^{*}
$$

where $C_{A}(S, \tau)$ is the price function of the non-callable American call. The above left hand side inequality is deduced from the property that "noncallable" corresponds to " $\rho=0$ ". For $\tau \leq \tau^{*}$, the callable American warrant is equivalent to its non-callable counterpart, hence the result in eq. (4.8). For $\rho=\infty$, the value function $V(S, \tau ; \infty)$ jumps from $C_{A}(S, \tau)$ to the rebate value $R$ when $\tau$ increases across $\tau^{*}$ and the optimal exercise curve is given by Eq. (4.7b). Here, $S_{\infty}^{*}(\tau)$ remains to be continuous at $\tau=\tau^{*}$ but there is a jump of discontinuity of $\frac{d S_{\infty}^{*}(\tau)}{d \tau}$ across $\tau=\tau^{*}$. For $\rho>0$ and when $\tau \leq \tau^{*}$, the pricing model does not contain the penalty term as $V \leq \psi$ for $\tau \leq \tau^{*}$. The penalty term $\rho \max (V-\psi, 0)$ enters into the pricing formulation [see Eq. (4.4a)] when $\tau$ increases beyond $\tau^{*}$. As the penalty term is finite when $\rho$ remains finite, we expect that $S_{\rho}^{*}(\tau)$ has a higher degree of smoothness across $\tau=\tau^{*}$ for finite value of $\rho$ as compared to its counterpart $S_{\infty}^{*}(\tau)$. It is thus conjectured that $S_{\rho}^{*}(\tau)$ has a continuous derivative at $\tau=\tau^{*}$ for finite $\rho$ value.

In Table 4, we show the numerical results of the American warrant value under varying values of stock price $S$ and penalty parameter $\rho$. In our numerical calculations, we use the same set of parameter values as those used to generate Figure 2. As expected, the callable American warrant value is an increasing function of the stock price $S$ but decreasing with respect to the intensity parameter $\rho$.

\section{Conclusion}

The prepayment right in a mortgage loan or the calling right in a callable security represents a pre-mature termination clause that places a cap on the market value of liabilities of the corresponding financial security. When 
the optimality of exercising the right is assumed, the pricing model of the derivative constitutes an optimal stopping problem. The optimal stopping rule and the price of the derivative can be obtained via the solution of the associated linear complementarity formulation. However, empirical studies have shown that these embedded rights are generally not executed optimally by the mortgagor or the security issuer. Suppose the propensity of mortgagor's prepayment or issuer's calling is modeled by the intensity of a Poisson process, we have shown that the pricing formulation resembles the penalty approximation approach for solving the linear complementarity formulation of an optimal stopping problem. The penalty parameter is seen to have a vivid financial interpretation. It can be visualized as the intensity of the Poisson process modeling the arrival of the mortgagor's prepayment or the issuer's calling.

For the mortgage loan valuation problem where prepayment is modeled as an event risk, the pricing of the value of liabilities becomes a non-linear problem. The boundary that separates the non-prepayment region and feasible prepayment region is obtained as part of the solution of the pricing model. We have obtained several theoretical results on the monotonicity properties of the separating boundary with respect to the intensity of prepayment and the level of transaction cost.

The pricing model of a callable American warrant can be formulated as a set of variational inequalities with double obstacle functions. More precisely, the value function is bounded from above by the payoff upon the issuer's calling and below by the payoff upon the holder's exercise. When the arrival of issuer's calling is modeled by a Poisson process, the pricing model of the callable American warrant resembles that of an American call option subject to the event risk of pre-mature termination. The double obstacle problem is simplified to a set of variational inequalities with one obstacle, except that one of the inequalities becomes non-linear. We have analyzed the optimal holder's exercise policy subject to risk of calling by the issuer. There exists a critical value of time to expiry below which the warrant is always in the non-calling region. When the warrant is subject to the risk of calling by the issuer, the optimal exercise price decreases with increasing value of intensity of calling.

We have proposed two versions of second order finite difference schemes for solving the penalty approximation of the linear complementarity formulation of an optimal stopping problem. With an appropriate discretization of the non-linear penalty term, our schemes exhibit quadratic rate of conver- 
gence even when constant sized time steps are used in the calculations. In one of our schemes, which takes the form of an explicit three-level scheme, enhanced computational efficiency is achieved since the Newton type of iterations at every time step are avoided.

As the final concluding remark, this paper shows how to model the mortgagor's prepayment in the mortgage loans and the issuer's call in callable derivatives as an event risk using the intensity based approach. We manage to link the pricing formulation that is based on the reduced form approach with the penalty approach in solving the linear complementarity formulation of the associated optimal stopping problem. Note that traded securities values may be affected by many other factors, like stochastic interest rates, liquidity and credit risk, and these factors have not been included in our proposed models. Our paper has not included empirical studies of the proposed models, say, implementing the calibration of the model parameters using traded market data. It would be interesting and useful to estimate the intensity using parameter estimation methods from traded data of mortgage securities and callable securities. However, before we perform full scale calibration exercises, it would be more preferable to construct comprehensive pricing models with inclusion of all essential factors that determine derivative prices. The empirical studies of such comprehensive pricing models will be relegated to future works.

\section{References}

[1] Carr, P. and V. Linetsky, 2000, "The valuation of executive stock options in an intensity-based framework," European Finance Review 4, 211-230.

[2] Dai, M. and Y.K. Kwok, 2007, "Optimal policies of call with notice period requirement," to appear in Asia-Pacific Financial Markets.

[3] Deng, Y., J.M. Quigley, and R. Van Order, 2000, "Mortgage terminations, heterogeneity and the exercise of mortgage options," Econometrica $68,275-307$.

[4] Forsyth, P.A. and K.R. Vetzal, 2002, "Quadratic convergence for valuing American options using a penalty method, "SIAM Journal on Scientific Computation 23, 2096-2123. 
[5] Friedman, A., 1982, Variational principles and free boundary problems, John Wiley and Sons, Inc.

[6] Goncharov, Y., 2006, "An intensity-based approach to the valuation of mortgage contracts and computation of the endogenous mortgage rate," International Journal of Theoretical and Applied Finance 9(6), 889-914.

[7] d'Halluin, Y., P.A. Forsyth and G. Labahn, 2004, "A penalty method for American options with jump diffusion processes", Numerische Mathematik 97, 321-352.

[8] Khaliq, A.Q.M., D.A. Voss and S.H.K. Kazmi, 2006, "A linearly implicit predictor-corrector scheme for pricing American options using a penalty method approach," Journal of Banking and Finance 30, 489-502.

[9] Kwok, Y.K. and L. Wu, 2000, "Effects of callable feature on early exercise feature," Review of Derivatives Research 4, 189-211.

[10] Lau, K.W. and Y.K. Kwok, 2004, "Anatomy of option features in convertible bonds," Journal of Futures Markets 24, 513-532.

[11] Lieberman, G.M., 1996, Second order parabolic differential equations, World Scientific, Singapore.

[12] McConnell, J.J., and M. Singh, 1994, "Rational prepayments and the valuation of collateralized mortgage obligations," Journal of Finance 49, 891-921.

[13] Schwartz, E.S., and W.N. Torous, 1992, "Prepayment, default, and the valuation of mortgage pass-through securities," Journal of Business 65, 221-239.

[14] Stanton, R., 1995, "Rational prepayment and the valuation of mortgage backed securities," Review of Financial Studies 8, 677-708. 
[15] Szimayer, A., 2004, "A reduced form model for ESO valuation," Mathematical Methods of Operations Research 59(1), 111-128.

[16] Szimayer, A., 2005, "Valuation of American options in the presence of event risk," Finance and Stochastics 9, 89-107.

[17] Thomas, J.W., 1995, Numerical partial differential equation: finite difference methods, Springer, Berlin.

[18] Zvan, R., P.A. Forsyth and K.R. Vetzal, 1998, "Penalty methods for American options with stochastic volatility," Journal of Computational and Applied Mathematics 91, 199-218. 


\section{Appendix A - Proof of Lemma 1}

Assume the contrary, that is, the open set $G=\left\{(r, t): L_{1}>L_{2}\right\}$ is nonempty. We then have

$$
\max \left(L_{1}-\psi, 0\right) \geq \max \left(L_{2}-\psi, 0\right) \quad \text { in } G \text {. }
$$

Consequently, we have

$$
\begin{aligned}
& \left(\frac{\partial}{\partial t}+\mathcal{L}\right)\left(L_{1}-L_{2}\right) \\
= & \rho_{1} \max \left(L_{1}-\psi, 0\right)-\rho_{2} \max \left(L_{2}-\psi, 0\right)+f_{2}-f_{1} \\
= & \rho_{1}\left[\max \left(L_{1}-\psi, 0\right)-\max \left(L_{2}-\psi, 0\right)\right]+\left(\rho_{1}-\rho_{2}\right) \max \left(L_{2}-\psi, 0\right) \\
& +\left(f_{2}-f_{1}\right) \geq 0 .
\end{aligned}
$$

On the boundary of $G$, we have $L_{1}-L_{2}=0$. By using the comparison principle of the linear operator $\left(\frac{\partial}{\partial t}+\mathcal{L}\right)$, we obtain $L_{1} \leq L_{2}$ [see Lieberman (1996)]. A contradiction is encountered, so we have the desired result.

\section{Appendix B - Proof of Lemma 2}

It is reasonable to assume $-\frac{d P(t)}{d t}+r P(t) \geq 0$ since the mortgage loan is amortized throughout the life of the contract. For $X_{1}<X_{2}$, it suffices to show

$$
L\left(r, t ; X_{1}\right)-P(t)\left(1+X_{1}\right) \geq L\left(r, t ; X_{2}\right)-P(t)\left(1+X_{2}\right)
$$

or equivalently

$$
L\left(r, t ; X_{1}\right) \geq L\left(r, t ; X_{2}\right)-P(t)\left(X_{2}-X_{1}\right)
$$

We define

$$
\widetilde{L}(r, t)=L\left(r, t ; X_{2}\right)-P(t)\left(X_{2}-X_{1}\right)
$$


It is easy to check that

$$
\begin{aligned}
\left(\frac{\partial}{\partial t}+\mathcal{L}\right) \widetilde{L}= & \rho \max \left(\widetilde{L}-P(t)\left(1+X_{1}\right), 0\right)-\left[c(t)+\lambda P(t)\left(1+X_{1}\right)\right] \\
& +\left(X_{2}-X_{1}\right)\left[-\frac{d P(t)}{d t}+r P(t)\right]
\end{aligned}
$$

By observing

$$
\left(X_{2}-X_{1}\right)\left[-\frac{d P(t)}{d t}+r P(t)\right] \geq 0
$$

and applying Lemma 1 , we infer that $\widetilde{L}(r, t) \leq L\left(r, t ; X_{1}\right)$. Hence, we obtain the desired result.

\section{Appendix C - Proof of convergence of Scheme one [see Eqs. $(3.3 \mathrm{a}, \mathrm{b})]$}

Let $\boldsymbol{U}^{n}=\left(U_{1}^{n} \cdots U_{N_{S}}^{n}\right)$ and $\boldsymbol{\Phi}=\left(\phi\left(S_{1}\right) \cdots \phi\left(S_{N_{S}}\right)\right)$, then Scheme One can be expressed as

$$
\boldsymbol{U}^{n+1}-\boldsymbol{U}^{n}+A \frac{\boldsymbol{U}^{n+1}+\boldsymbol{U}^{n}}{2}=\Lambda^{n+\frac{1}{2}}\left(\boldsymbol{\Phi}-\frac{\boldsymbol{U}^{n+1}+\boldsymbol{U}^{n}}{2}\right),
$$

where $A$ is the matrix representing the difference operator $\mathcal{L}_{h}$ and $\Lambda^{n+\frac{1}{2}}$ is a diagonal matrix whose diagonal entry $\Lambda_{j j}^{n+\frac{1}{2}}$ is

$$
\Lambda_{j j}^{n+\frac{1}{2}}= \begin{cases}\rho \Delta \tau & \text { if } \phi\left(S_{j}\right)>\frac{U_{j}^{n+1}+U_{j}^{n}}{2}, \quad j=1,2, \cdots, N_{S} . \\ 0 & \text { otherwise }\end{cases}
$$

To establish the convergence of the above numerical scheme, it is necessary to show that $\boldsymbol{U}^{n}$ satisfies the following set of discrete variational inequalities 
(Forsyth and Vetzal, 2002):

$$
\begin{aligned}
& \boldsymbol{U}^{n+1}-\boldsymbol{U}^{n}+A\left(\frac{\boldsymbol{U}^{n+1}+\boldsymbol{U}^{n}}{2}\right) \geq 0 \\
& \quad \frac{\boldsymbol{U}^{n+1}+\boldsymbol{U}^{n}}{2}-\boldsymbol{\Phi} \geq-\frac{C}{\rho \Delta \tau} \\
& {\left[\boldsymbol{U}^{n+1}-\boldsymbol{U}^{n}+A\left(\frac{\boldsymbol{U}^{n+1}+\boldsymbol{U}^{n}}{2}\right)=0\right] V\left(\left|\frac{\boldsymbol{U}^{n+1}+\boldsymbol{U}^{n}}{2}-\boldsymbol{\Phi}\right| \leq \frac{C}{\rho \Delta \tau}\right),}
\end{aligned}
$$

where $C$ is a positive constant independent of $\rho \Delta \tau, \Delta \tau$ and $\Delta S$.

To establish the satisfaction of the above set of inequalities, it suffices to show that the following quantity

$$
\Lambda^{n+\frac{1}{2}}\left(\boldsymbol{\Phi}-\frac{\boldsymbol{U}^{n+1}+\boldsymbol{U}^{n}}{2}\right)
$$

is bounded, independent of $\rho \Delta \tau, \Delta \tau$ and $\Delta S$. Let $k$ denote the node at which the penalty term achieves its maximum value, we can infer that

$$
\Phi_{k}-\frac{U_{k}^{n+1}+U_{k}^{n}}{2} \geq 0
$$

Applying the discrete maximum principle, we deduce that

$$
A\left(\Phi_{k}-\frac{U_{k}^{n+1}+U_{k}^{n}}{2}\right) \geq 0 \quad \text { or } \quad A\left(\frac{U_{k}^{n+1}+U_{k}^{n}}{2}\right) \leq A \Phi_{k} .
$$

Since Forsyth and Vetzal (2002) have established $\|A \Phi\|_{\infty} \leq O\left(\frac{\Delta \tau}{\Delta S}\right)$, so we have

$$
A\left(\frac{U_{k}^{n+1}+U_{k}^{n}}{2}\right) \leq O\left(\frac{\Delta \tau}{\Delta S}\right)
$$

Now, we rewrite Eq. (C.1) as

$$
\left(I+\frac{A}{2}\right) \boldsymbol{U}^{n+1}=\left(I-\frac{A}{2}\right) \boldsymbol{U}^{n}+\Lambda^{n+\frac{1}{2}}\left(\boldsymbol{\Phi}-\frac{\boldsymbol{U}^{n+1}+\boldsymbol{U}^{n}}{2}\right) .
$$

Suppose the time step $\Delta \tau$ is chosen such that all elements of $I-\frac{A}{2}$ are nonnegative, thus the right hand side of Eq. (C.4) is non-negative. Since $I+\frac{A}{2}$ 
is also a $M$-matrix. Recall that $M$-matrix is a diagonally dominant matrix with positive diagonals and non-positive off diagonals. Since the inverse of an $M$-matrix is non-negative, we then deduce that

$$
\boldsymbol{U}^{n} \geq 0 \text { for all } n \text {. }
$$

As a remark, the requirement that $I+\frac{A}{2}$ and $I-\frac{A}{2}$ being $M$-matrices is meant to give sufficiency for convergence. They are not necessary for convergence in actual calculations.

Together with Eq. (C.2), we can infer that

$$
U_{k}^{n+1} \leq 2\|\Phi\|_{\infty}
$$

Lastly, we combine Eqs. (C.1), (C.3), (C.6) together to obtain

$$
O \leq\left[\Lambda^{n+\frac{1}{2}}\left(\boldsymbol{\Phi}-\frac{\boldsymbol{U}^{n+1}+\boldsymbol{U}^{n}}{2}\right)\right]_{k} \leq 2\|\boldsymbol{\Phi}\|_{\infty}+O\left(\frac{\Delta \tau}{\Delta S}\right)
$$

so that

$$
\left\|\Lambda^{n+\frac{1}{2}}\left(\boldsymbol{\Phi}-\frac{\boldsymbol{U}^{n+1}+\boldsymbol{U}^{n}}{2}\right)\right\|_{\infty} \leq K
$$

where $K$ is independent of $\rho \Delta \tau, \Delta \tau$ and $\Delta S[$ provided that we choose $\Delta \tau$ and $\Delta S$ such that $\left.\frac{\Delta \tau}{\Delta S}=O(1)\right]$. 


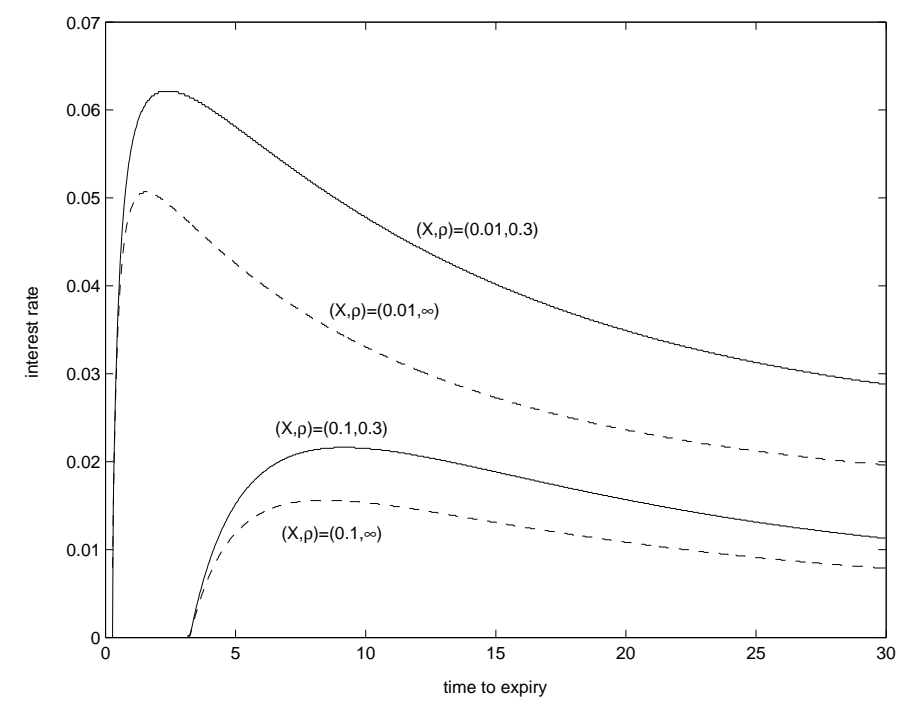

Figure 1 Plot of the separating boundary $r^{*}$ against time to expiry $T-t$ with varying values of the transaction cost factor $X$ and penalty parameter $\rho$. With a higher value of $\rho$ or $X, r^{*}$ assumes a lower value.

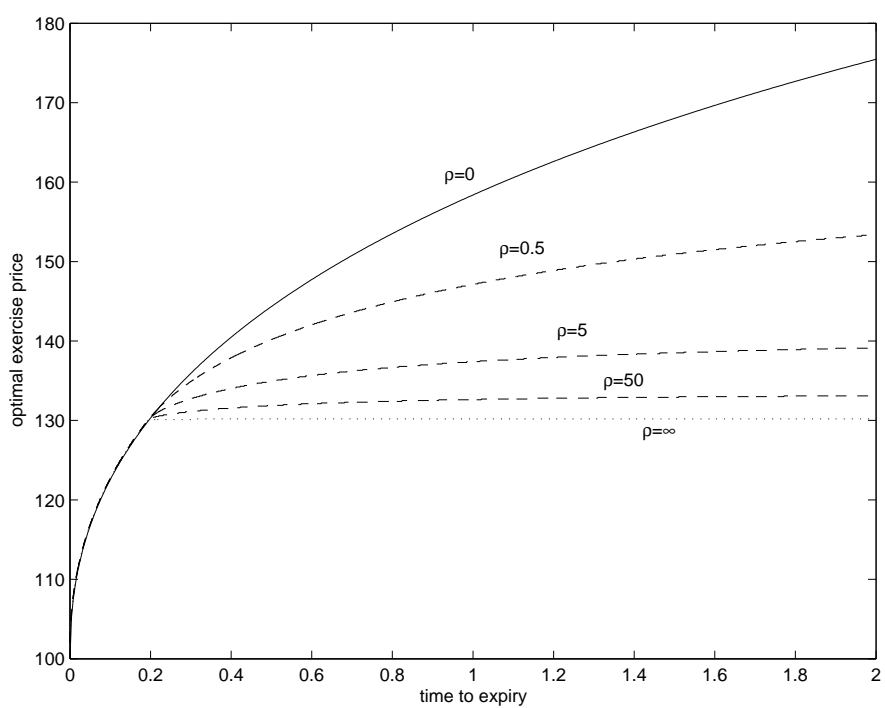

Figure 2 Plot of the optimal exercise price $S^{*}$ against time to expiry $T-t$ with varying values of the intensity $\rho$. With a higher value of $\rho, S^{*}$ assumes a lower value. 


\begin{tabular}{|l|l|l|l|l|l|l|l|l|}
\hline \multicolumn{2}{|c|}{ Scheme One } & \multicolumn{3}{|c|}{ Forsyth-Vetzal's scheme } \\
\hline$N_{\tau}$ & $N_{S}$ & Value & Change & Ratio & Value & Change & Ratio \\
\hline \multicolumn{8}{|c|}{$\sigma=0.2$, spatial domain of computation = $[0,200]$} \\
\hline 100 & 200 & 3.06908 & & & 3.06751 & & \\
\hline 200 & 400 & 3.06986 & 0.00078 & & 3.06910 & 0.00159 & \\
\hline 400 & 800 & 3.07005 & 0.00019 & 4.1 & 3.06968 & 0.00058 & 2.8 \\
\hline 800 & 1600 & 3.07010 & 0.00005 & 4.1 & 3.06991 & 0.00023 & 2.5 \\
\hline 1600 & 3200 & 3.07010 & 0.00001 & 4.3 & 3.07001 & 0.00010 & 2.3 \\
\hline \multicolumn{8}{|c|}{$\sigma=0.8$, spatial domain of computation = 0,1000$]$} \\
\hline 100 & 200 & 14.67638 & & & 14.67431 & & \\
\hline 200 & 400 & 14.67826 & 0.00188 & & 14.67729 & 0.00298 & \\
\hline 400 & 800 & 14.67873 & 0.00047 & 4.0 & 14.67828 & 0.00099 & 3.0 \\
\hline 800 & 1600 & 14.67885 & 0.00012 & 4.0 & 14.67863 & 0.00035 & 2.8 \\
\hline 1600 & 3200 & 14.67887 & 0.00002 & 4.2 & 14.67877 & 0.00014 & 2.5 \\
\hline
\end{tabular}

Table 1 Comparison of the convergence behaviors of pricing calculations of an American put option using Scheme One and Forsyth-Vetzal's scheme using constant sized time steps. The numerical results obtained from Scheme One demonstrate quadratic rate of convergence.

\begin{tabular}{|c|c|c|c|c|c|c|c|}
\hline \multicolumn{2}{|c|}{} & \multicolumn{2}{|c|}{ Stanton's Scheme } & \multicolumn{2}{c|}{ Scheme One } & \multicolumn{2}{c|}{ Scheme Two } \\
\hline$N_{\tau}$ & $N_{S}$ & mean squared errors & ratio & mean squared errors & ratio & mean squared errors & ratio \\
\hline 200 & 200 & 13.468 & - & 0.062315 & - & 0.038450 & - \\
\hline 400 & 400 & 6.706 & 2.0 & 0.011925 & 5.2 & 0.009535 & 4.0 \\
\hline 800 & 800 & 3.347 & 2.0 & 0.002557 & 4.7 & 0.002374 & 4.0 \\
\hline 1600 & 1600 & 1.672 & 2.0 & 0.000596 & 4.3 & 0.000591 & 4.0 \\
\hline 3200 & 3200 & 0.835 & 2.0 & 0.000146 & 4.1 & 0.000147 & 4.0 \\
\hline
\end{tabular}

Table 2 Examination of the rate of convergence of numerical calculations of pricing a mortgage loan using Stanton's scheme and our proposed modified Crank-Nicolson schemes. 


\begin{tabular}{|c|c|c|c|c|c|c|}
\hline \multicolumn{2}{|c|}{} & \multicolumn{5}{|c|}{$\rho$} \\
\hline$X$ & $\lambda$ & 0.03 & 0.3 & 3 & 30 & $\infty$ \\
\hline 0.01 & 0 & 0.9900 & 0.9885 & 0.9853 & 0.9839 & 0.9836 \\
& 0.05 & 1.0402 & 1.0330 & 1.0174 & 1.0110 & 1.0100 \\
& 0.5 & 1.0687 & 1.0565 & 1.0252 & 1.0119 & 1.0100 \\
\hline 0.1 & 0 & 0.9903 & 0.9903 & 0.9903 & 0.9902 & 0.9902 \\
& 0.05 & 1.0732 & 1.0730 & 1.0721 & 1.0714 & 1.0713 \\
& 0.5 & 1.1501 & 1.1405 & 1.1141 & 1.1019 & 1.1000 \\
\hline
\end{tabular}

Table 3 Liability value of a mortgage loan under varying values of transaction cost factor $X$, intensity of exogenous prepayment $\lambda$ and penalty parameter $\rho$.

\begin{tabular}{|c|c|c|c|c|c|c|}
\hline & \multicolumn{6}{|c|}{$\rho$} \\
\hline$S$ & 0 & 0.05 & 0.5 & 5 & 50 & $\infty$ \\
\hline 100 & 14.6100 & 14.5641 & 14.3595 & 13.9458 & 13.6514 & 13.4675 \\
120 & 27.1556 & 26.9832 & 26.3632 & 25.2268 & 24.4795 & 24.0329 \\
140 & 42.9089 & 42.4547 & 41.1993 & 40 & 40 & 40 \\
\hline
\end{tabular}

Table 4 Callable American warrant value under varying values of stock price $S$ and intensity parameter $\rho$. 